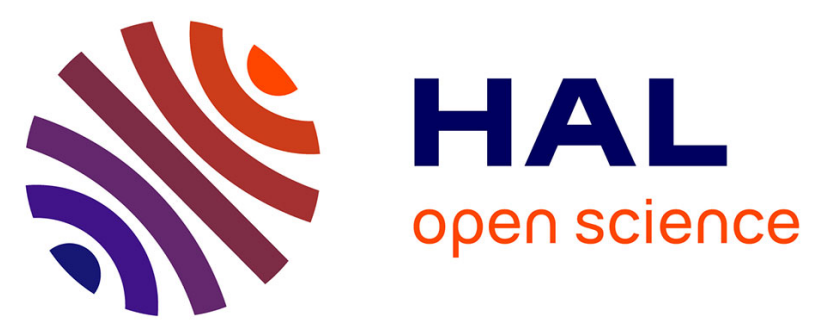

\title{
Comment structurer la complexité sans renoncer à l'exhaustivité thématique d'un état de l'art en sciences de gestion? Retour d'expérience sur l'apport méthodologique des outils de classification de données qualitatives
}

Céline Averseng

\section{To cite this version:}

Céline Averseng. Comment structurer la complexité sans renoncer à l'exhaustivité thématique d'un état de l'art en sciences de gestion? Retour d'expérience sur l'apport méthodologique des outils de classification de données qualitatives. Revue management \& avenir, 2011, 41 (1), pp.369. 10.3917/mav.041.0369 . hal-01992760

\author{
HAL Id: hal-01992760 \\ https://hal.science/hal-01992760
}

Submitted on 24 Jan 2019

HAL is a multi-disciplinary open access archive for the deposit and dissemination of scientific research documents, whether they are published or not. The documents may come from teaching and research institutions in France or abroad, or from public or private research centers.
L'archive ouverte pluridisciplinaire HAL, est destinée au dépôt et à la diffusion de documents scientifiques de niveau recherche, publiés ou non, émanant des établissements d'enseignement et de recherche français ou étrangers, des laboratoires publics ou privés. 


\title{
Comment structurer la complexité sans renoncer à l'exhaustivité thématique d'un état de l'art en sciences de gestion? Retour d'expérience sur l'apport méthodologique des outils de classification de données qualitatives.
}

\section{How to structure the complexity without giving up the thematic exhaustiveness of a state of the art in management science? Feedback on the methodological contribution of classification tools of qualitative data.}

\author{
AVERSENG CELINE \\ PRAG-DOCTORANT \\ CREGOR \\ IAE de Montpellier, \\ Université de Montpellier II \\ celine.averseng@univ-montp2.fr
}

IAE, Place Eugène Bataillon, 34095 MONTPELLIER

Tél : 0467149333

Fax : 0467144242

PRAG (Professeur Agrégée), responsable pédagogique du Master B\&A (Master Banque et Assurances par apprentissage) et de la Préparation à l'Agrégation Economie et Gestion.

Domaines de compétence : Système d'information (ERP), comptabilité et contrôle de gestion, recherche opérationnelle, stratégie, économie. 


\section{Comment structurer la complexité sans renoncer à l'exhaustivité thématique d'un état de l'art en sciences de gestion? Retour d'expérience sur l'apport méthodologique des outils de classification de données qualitatives.}

\section{How to structure the complexity without giving up the thematic exhaustiveness of a state of the art in management science? Feedback on the methodological contribution of classification tools of qualitative data.}

\section{$\square$ Résumé}

L'état de l'art est une étape indispensable dans un processus de recherche. L'auteur présente un retour d'expérience sur la démarche qui a été la sienne en la matière. L'objectif est ici d'apporter des éléments de réponse et des pistes de réflexion à la question suivante : Comment structurer la complexité sans renoncer à l'exhaustivité thématique d'un état de l'art en sciences de gestion ? La démarche utilisée présente l'originalité de montrer comment plusieurs applications informatiques peuvent être combinées afin de rationaliser le travail de documentation, d'analyse et de synthèse que le chercheur devra effectuer.

Mots clefs : état de l'art, application informatique, carte heuristique, analyse thématique, organisation documentaire, analyse qualitative.

\section{Abstract}

The state of the art is an indispensable step in a research process. The author presents a feedback on his approach in the matter. The objective here is to provide some answers and tracks of reflection to the following question: How to structure the complexity without giving up the thematic exhaustiveness of a state of the art in management science? The used approach presents the originality to show how several computing applications can be combined to rationalize the work of documentation, analysis and synthesis which the researcher will have to make.

Keywords: state of the art, computing tools, mind map, thematic analysis, documentary organization, qualitative analysis. 


\section{Introduction}

Les sources documentaires dont les chercheurs disposent aujourd'hui sont pléthoriques. Leurs formats se multiplient et leur numérisation ouvre de nouvelles opportunités en termes d'accès à la connaissance par tous, à distance et à n'importe quel moment. Cela ne va pas sans poser un certain nombre de problèmes : face au volume des corpus théoriques qui doivent être étudiés et mobilisés dans le cadre d'une recherche, le chercheur peut éprouver des difficultés à identifier les grandes thématiques et problématiques de son objet d'étude. Comment s'y retrouver dans la masse de documents à laquelle il a accès ? Comment organiser, matériellement, cet ensemble de sources ? Comment extraire les informations qui auront par la suite une incidence fondamentale sur le travail réalisé ? Comment retrouver une référence, identifiée à un moment donné du processus de recherche ? Enfin, comment structurer, organiser, les éléments recueillis, afin d'en faire un ensemble cohérent sur lequel la démonstration du chercheur pourra se fonder?

L'objet de cette communication est d'apporter quelques pistes de réflexion à ces nombreuses interrogations que le chercheur ne manquera pas de se poser dans son processus de recherche. Elle a pour but de montrer l'apport des outils de classification des données face à la profusion des sources bibliographiques, afin de répondre à la question de recherche suivante : Comment structurer la complexité sans renoncer à l'exhaustivité thématique d'un état de l'art en sciences de gestion ?

Il s'agit ici de rendre compte d'un retour d'expérience, dans lequel le rôle des outils informatiques a été déterminant. Cette communication s'inscrit dans le cadre d'un travail de recherche portant sur le management des processus dans une entité industrielle. Elle est le fruit d'un long travail d'analyse, d'exploration et d'utilisation de différents outils informatiques qui sont à la disposition du chercheur en sciences de gestion.

Nous avons souhaité mettre en évidence une démarche originale, fondée sur l'utilisation d'un certain nombre d'outils. Etant donné la complexité du sujet mise en évidence par les entretiens exploratoires, nous avons souhaité mettre en place une méthode de travail très structurée afin d'analyser la littérature. Les outils mobilisés ont permis au chercheur d'établir un lien entre le travail d'analyse des données collectées sur le terrain d'une part, et l'étude de la bibliographie sur le sujet d'étude d'autre part, à savoir le management des processus : dans les deux cas, la démarche utilisée pour analyser les informations est sensiblement la même.

Cette analyse de la littérature s'est faite en neuf phases successives (dont la synthèse est présentée dans la Figure 1), que nous pourrons regrouper en trois grandes thématiques : la gestion, l'analyse et la synthèse des sources documentaires. Dans ce cadre, cinq applications informatiques distinctes ont été mobilisées : Microsoft ${ }^{\circledR}$ OneNote 2007, EndNote Web, FreeMind 0.8.1, QSR ${ }^{\circledR}$ NVivo 7 et Microsoft ${ }^{\circledR}$ Word 2007. 


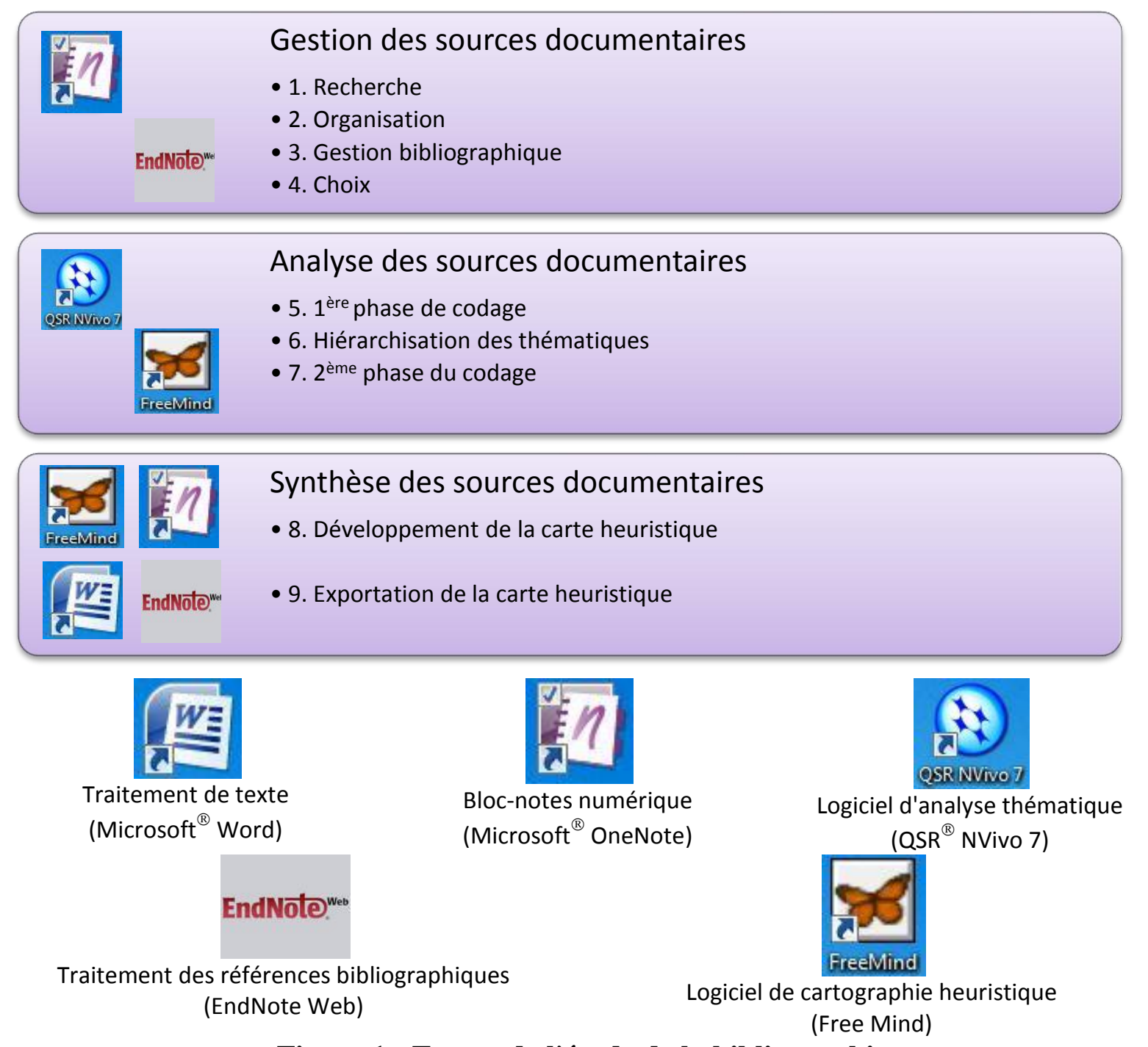

Figure 1 : Etapes de l'étude de la bibliographie

Nous traiterons alors dans une $1^{\text {ère }}$ partie de la gestion des sources documentaires, dans une seconde, de leur analyse, et dans une troisième de leur synthèse. Les apports des différentes applications informatiques seront abordés tout au long de ces trois parties. Enfin, un quatrième point proposera une discussion sur l'ensemble de la démarche adoptée.

\section{Gestion des sources documentaires}

La gestion des sources documentaires qui devra être effectuée par le chercheur est loin d'être une évidence. Le nombre très important des sources soulève de nombreuses difficultés que certaines applications informatiques permettent de contourner. Une organisation pertinente de ces sources présente pourtant de multiples avantages : un accès plus rapide aux documents réunis, l'annotation de ces derniers, mais aussi leur citation dans le travail de production du chercheur (rédaction de communications, articles, thèses...). Outre ces avantages, qui offrent des gains de temps non négligeables, une telle organisation pourra aussi permettre la mise en évidence de références jugées significatives du domaine étudié : le chercheur obtiendra ainsi un point de départ pour effectuer son état de l'art.

La démarche du chercheur devra alors être méthodique : les sources documentaires devront être recherchées (point 1.1) et organisées (point 1.2). Elles pourront ensuite faire l'objet d'une 
gestion bibliographique (point 1.3) et certaines d'entre elles, jugées significatives dans le domaine étudié, pourront être identifiées (point 1.4).

\subsection{Recherche des sources documentaires}

Une grande partie des articles qui ont été utilisés dans le cadre de cette recherche sont en format numérique, en raison du caractère relativement récent du sujet traité (postérieur aux années 1990) : une centaine d'articles ont été "collectés" dans cette première phase. Des articles et ouvrages en format papier ont aussi été utilisés, soit via une numérisation, si leur taille était raisonnable, soit via l'utilisation d'un système de référencement et de renvois.

La principale difficulté ne réside pas dans l'obtention de ces documents (les abonnements et bases de données en lignes sont nombreux et de grande qualité), mais dans leur organisation matérielle, afin qu'ils puissent être utilisés ultérieurement : impressions, annotations manuelles et classement dans des classeurs ou autres pochettes peuvent atteindre très rapidement leurs limites.

\subsection{Organisation des sources documentaires}

Une des premières difficultés rencontrées par le chercheur suite à cette phase de recherche documentaire consiste à organiser matériellement les éléments réunis. Or la plupart des documents numériques ne peuvent être annotés directement (même si des outils tels que les tablettes graphiques ou les tablet $\mathrm{PC}^{1}$ offrent de nombreuses possibilité en la matière, en alliant source numérique et saisie manuscrite) et la gestion de fichiers distincts s'avère rapidement fastidieuse : le premier contenant un article, par exemple, et le second les annotations concernant ce dernier. Pour reprendre l'exemple précédent, comment faciliter l'accès aux articles, accéder à leur référence complète (dans une optique de recherche ultérieure et de mobilisation dans le cadre d'une production scientifique), et enfin les annoter?

Certaines applications numériques permettent une organisation documentaire particulièrement utile pour le chercheur ; c'est le cas des outils qualifiés de "bloc-notes numérique", même si le choix de cette qualification reflète relativement mal les nombreuses possibilités offertes par ces applications. Elles permettent en effet de classer, d'accéder facilement aux sources (articles, extraits d'ouvrage, liens numériques) et à leurs références (via le classement mis en place par l'utilisateur ou via le moteur de recherche intégré à l'outil), mais aussi de les annoter avec une grande liberté et de mettre en évidence les points (extraits, citations, illustrations...) susceptibles d'être réutilisés : Figure 2 (le logiciel utilisé dans l'étude est Microsoft ${ }^{\circledR}$ OneNote $\left.2007^{2}\right)$.

Un bloc-note numérique est composé de sections (« Thèse », «Processus », « Biblio » etc... dans la Figure 2), elles mêmes subdivisées en pages («Davenport», «Hammer »...). Les sections permettront d'organiser les sources en grandes thématiques, au gré des besoins du chercheur. Les pages pourront être créées pour les sous thématiques, ou pour regrouper des éléments. Elles permettent d'agencer librement des informations qui pourront prendre des formes diverses : texte, image, tableau, mais aussi indicateurs (qui pourront être utilisés pour souligner l'importance de tel ou tel point), liens hypertextes et raccourcis vers des fichiers, sous forme d'icones (« La gestion par processus...» dans notre exemple). Une impression du

\footnotetext{
${ }^{1}$ Les Tablet PC sont des ordinateurs portables dont l'écran est tactile. L'utilisateur peut donc écrire directement, de façon manuscrite sur des documents numérique de divers formats.

${ }^{2}$ L'équivalent en logiciel libre de Microsoft ${ }^{\circledR}$ OneNote 2007est BasKet Note Pads.
} 
fichier dans une page est aussi possible : l'article peut alors faire l'objet d'annotations directes (insertion de commentaires écrits ou possibilité de surligner le texte).

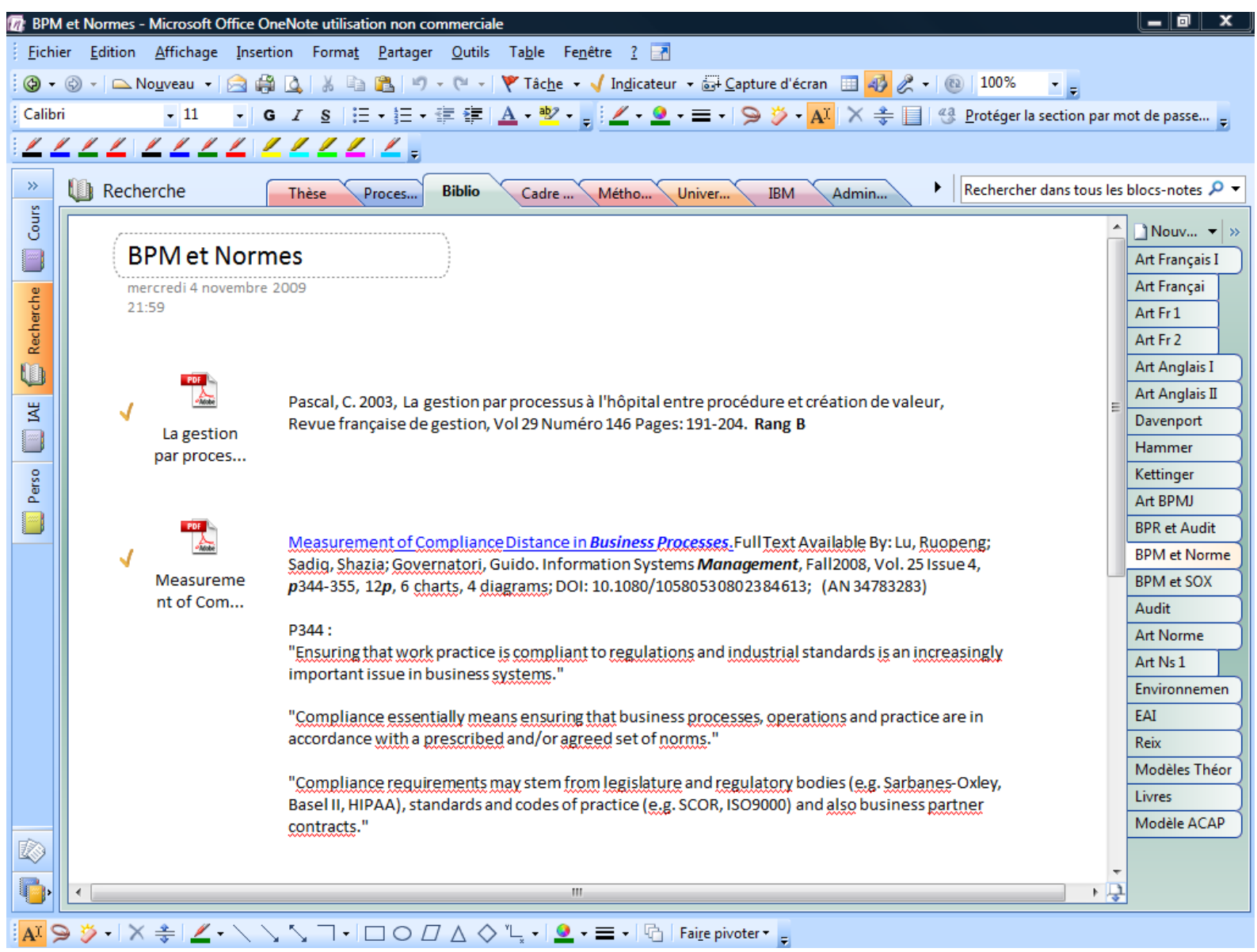

Figure 2 : Bloc-notes numérique (Microsoft ${ }^{\circledR}$ OneNote 2007)

Ce type d'outil permettra ainsi d'accéder plus facilement aux sources, via notamment un classement thématique et la mise en place de liens. Cependant, il n'offre pas l'opportunité de gérer de façon synthétique (ou tout simplement alphabétique) les références des sources en questions, contrairement aux applications de gestion bibliographique : point suivant.

\subsection{Gestion bibliographique des sources documentaires}

Les logiciels et/ou applications en ligne de gestion bibliographique tels que EndNote, EndNote $\mathrm{Web}^{3}$ (Figure 3) ou encore Zotero ${ }^{4}$ permettent de constituer une base de données personnelle des références utilisées par le chercheur. La plupart des abonnements électroniques mis à dispositions des chercheurs par leurs institutions permettent d'exporter directement les références dans ces applications ; c'est le cas par exemple des abonnements accessibles via Business Source Premier, ou ABI/inform. Il est aussi possible d'en effectuer une saisie directe ou via l'identifiant $\mathrm{DOI}^{5}$, ISSN ${ }^{6}$ ou ISBN ${ }^{7}$. L'usage d'indicateurs (symboles) dans le bloc-notes

${ }^{3}$ http://www.endnoteweb.com/

${ }^{4}$ http://www.zotero.org/

${ }^{5}$ Digital Object Identifier ("identifiant d'objet numérique") : mécanisme d'identification de ressources numérisées

${ }^{6}$ International Standard Serial Number : numéro international qui permet d'identifier de manière unique une publication en série (pour les journaux, périodiques) 
numérique permettra alors de pointer les articles ayant été enregistrés dans le gestionnaire de bibliographie : l'objectif est d'ici d'éviter les saisies redondantes et les oublis.

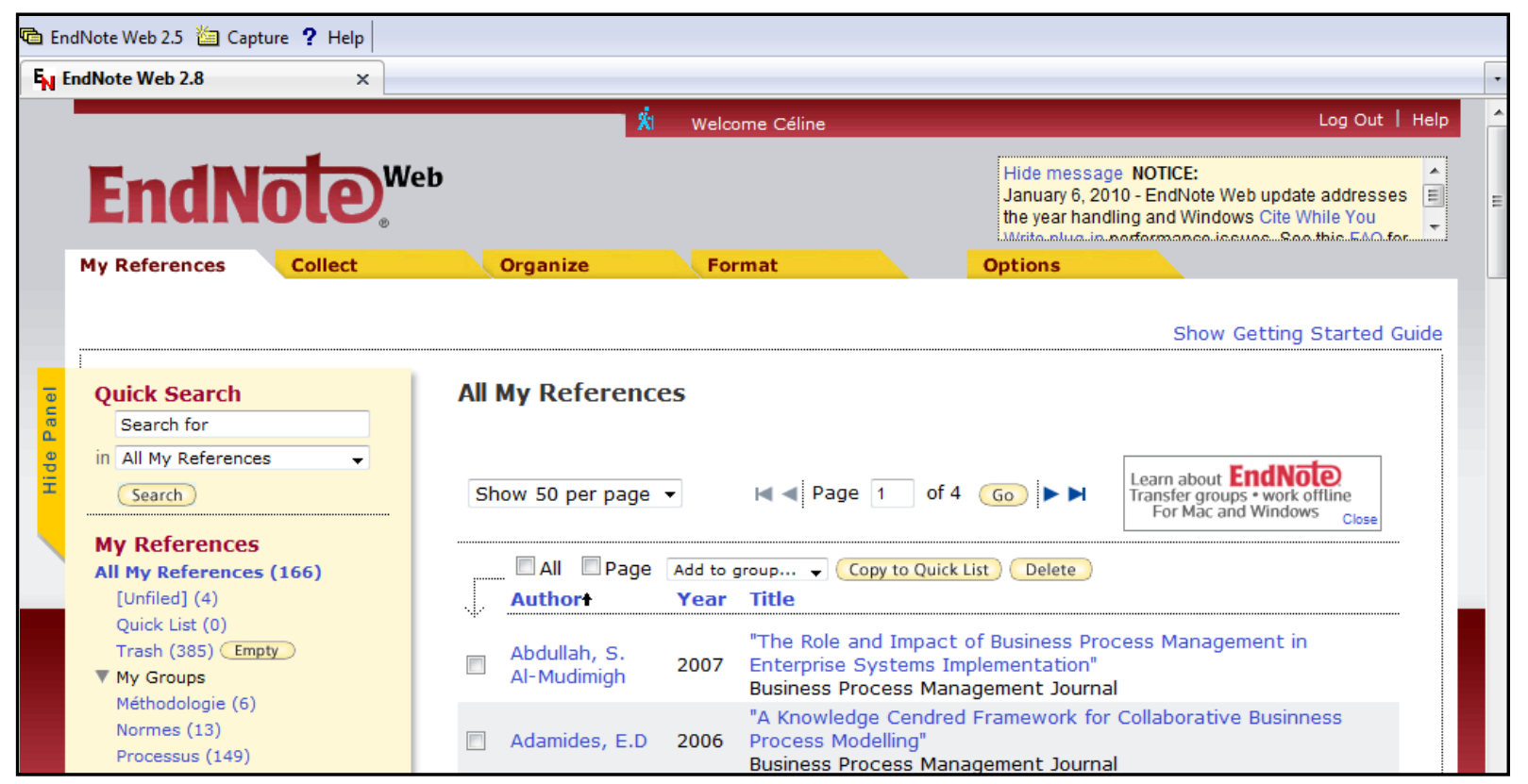

Figure 3 : Traitement des références bibliographiques (EndNote Web)

L'intérêt majeur de ces applications est la possibilité qu'elles offrent d'insérer directement les références bibliographiques que le chercheur souhaite mobiliser dans un traitement de texte ${ }^{8}$ : Figure 4. L'outil propose la liste des références enregistrées dans EndNote Web, permet leur insertion dans le corps du texte et crée la bibliographie automatiquement au fur et à mesure de la rédaction.

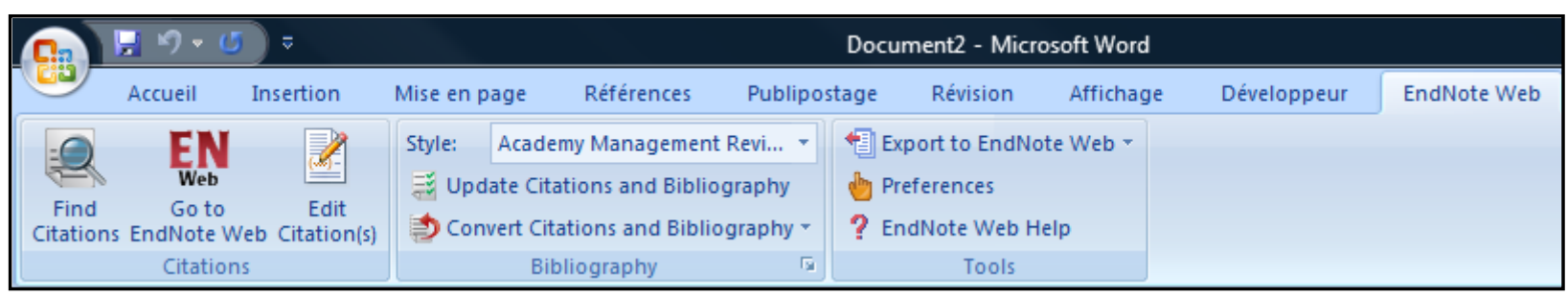

Figure 4 : Module supplémentaire EndNoteWeb dans Microsoft ${ }^{\circledR}$ Word

Le gain de temps est alors particulièrement significatif, au niveau de la recherche et de l'insertion des références dans le document, mais aussi de leur mise en forme et de l'établissement de la bibliographie : l'application propose un très grand nombre de modèles de mise en forme utilisés par la plupart des grandes revues internationales, dont un exemple est proposé en Figure 5 .

${ }^{7}$ International Standard Book Number (numéro international normalisé du livre): numéro international qui permet d'identifier de façon unique chaque livre publié.

${ }^{8}$ L'application en ligne EndNoteWeb permet de rajouter un module dans le logiciel Microsoft ${ }^{\circledR}$ Word. Ce module permettra de "puiser" directement les éléments dans la base de données en ligne des références bibliographiques et de les insérer dans le texte, via la saisie d'un mot clé, du nom de l'auteur et/ou d'une date. 
Adamides, E. D., \& Karacapilidis, N. 2006. "A Knowledge Cendred Framework for Collaborative Business Process Modelling", Business Process Management Journal, Vol. 12 (5): 557-575.

Aguilar-Saven, R. S. 2004. "Business Process Modelling: Review and Framework". International Journal of Production Economics, 90(2): 129-149.

Ayachi Ghannouchi, S., \& Ghannouchi, S.-E. 2008. "Une expérience de BPR dans un hôpital tunisien". Systèmes d'Information et Management, 13(1): 89.

Figure 5 : Exemple de mise en forme automatique de la bibliographie

(modèle de l'Academy Management Review)

\subsection{Choix d'un nombre réduit d'articles jugés significatifs}

A ce stade du processus de recherche, le chercheur dispose de sources documentaires organisées, dont certaines sont annotées. L'objectif de ce dernier est l'identification des grandes notions et thématiques de son domaine d'étude mais, dans cette optique, il va devoir rapidement faire face à une grande quantité d'annotation, et même si le moteur de recherche du bloc-notes numérique permet d'extraire les informations via l'utilisation de mots clés ou d'indicateurs, il atteint rapidement ses limites. Comment identifier les grands concepts contenus dans les articles académiques afin de pouvoir en présenter une synthèse ?

Notre point de départ a consisté à identifier un nombre réduit d'articles jugés significatifs du domaine d'étude. Leur choix a été effectué en fonction de leur titre et résumé, auteur (auteurs fondateurs du domaine ${ }^{9}$ ), revue, ou encore, le cas échéant, en fonction des mots clés les plus récurrents. Des applications en ligne telles que Wordle ${ }^{10}$, ont aussi permis d'obtenir très rapidement des représentations graphiques fondées sur la mise en évidence des mots les plus utilisés dans un texte : Cf. Figure 6. De telles représentations ont pu compléter les informations issues des résumés, mots clés et d'une première lecture rapide des articles.

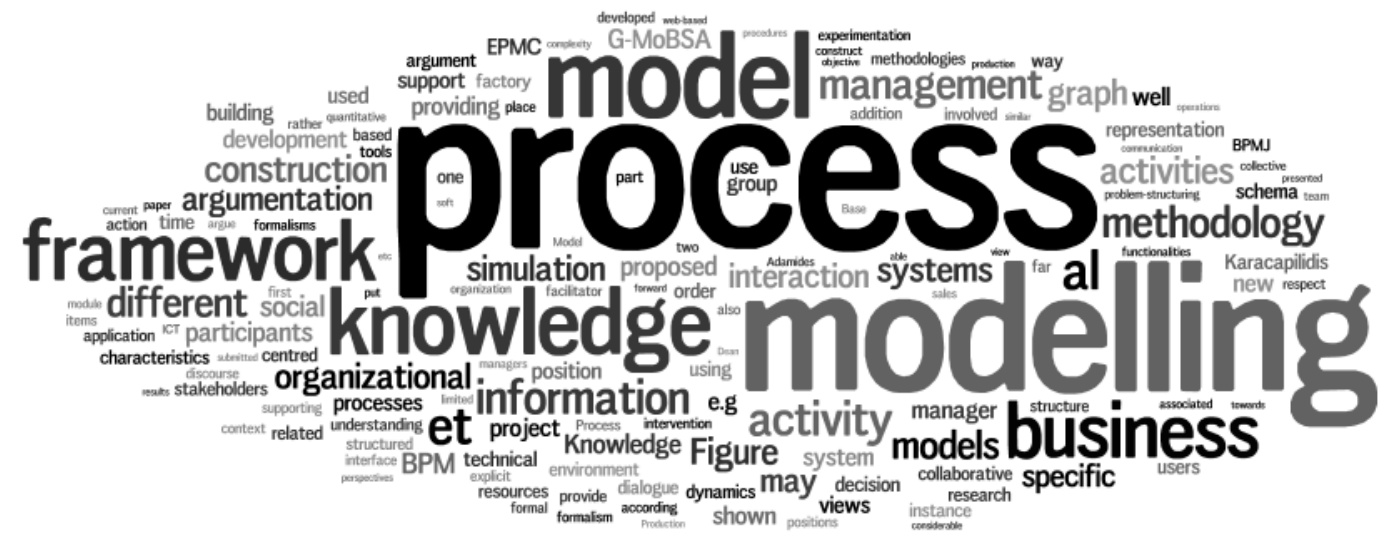

Figure 6 : Exemple de nuage de mots obtenu avec Wordle à partir de l'article d'Adamides et Karacapilidis (2006)

\footnotetext{
${ }^{9}$ Auteurs qui sont à l'origine des travaux sur le domaine étudié et qui y ont très largement contribué

${ }^{10}$ www.wordle.net
} 
La combinaison de ces différents critères nous a permis d'affiner la sélection des articles qui constitueront le point de départ de l'analyse. Notre choix s'est porté sur une quinzaine d'articles : Tableau 1.

\begin{tabular}{|c|c|c|c|c|c|}
\hline Auteur & Année & Revue & $\begin{array}{l}\text { Nombre } \\
\text { de pages }\end{array}$ & $\begin{array}{c}\text { Revue } \\
\text { clas- } \\
\text { sée }^{11}\end{array}$ & $\begin{array}{l}\text { Auteur } \\
\text { fondateur }\end{array}$ \\
\hline $\begin{array}{l}\text { Adamides, E. D., \& Kara- } \\
\text { capilidis, N. }\end{array}$ & 2006 & $\begin{array}{l}\text { Business Process Management } \\
\text { Journal }\end{array}$ & 18 & & \\
\hline Aguilar-Saven, R. S. & 2004 & $\begin{array}{l}\text { International Journal of Produc- } \\
\text { tion Economics }\end{array}$ & 20 & $A+/ 1$ & \\
\hline $\begin{array}{l}\text { Aytulun, S. K., \& Guneri, } \\
\text { A. F. }\end{array}$ & 2008 & $\begin{array}{l}\text { International Journal of Produc- } \\
\text { tion Research }\end{array}$ & 21 & $A / 2$ & \\
\hline $\begin{array}{l}\text { Bandara, W., \& Rose- } \\
\text { mann, M. }\end{array}$ & 2005 & $\begin{array}{l}\text { Systèmes d'Information et Ma- } \\
\text { nagement }\end{array}$ & 21 & $A / 2$ & \\
\hline Chan, S., \& Choi, C. & 1997 & $\begin{array}{l}\text { International Journal of Produc- } \\
\text { tion Economics }\end{array}$ & 12 & $\mathrm{~A}+/ 1$ & \\
\hline $\begin{array}{l}\text { Chatha, K. A., Ajaefobi, J. } \\
\text { O., \& Weston, R. H. }\end{array}$ & 2007 & $\begin{array}{l}\text { International Journal of Produc- } \\
\text { tion Research }\end{array}$ & 38 & $A / 2$ & \\
\hline $\begin{array}{l}\text { Davenport, T. H., \& Beers, } \\
\text { M. C. }\end{array}$ & 1995 & $\begin{array}{l}\text { Journal of Management Infor- } \\
\text { mation System }\end{array}$ & 23 & $\mathrm{~A}+/ 1$ & $\checkmark$ \\
\hline $\begin{array}{l}\text { Dennis, A. R., Carte, T. A., } \\
\text { \& Kelly, G. G. }\end{array}$ & 2003 & Decision Support Systems & 27 & $A / 2$ & \\
\hline Hammer, M. & 1990 & Harvard Business Review & 8 & & $\checkmark$ \\
\hline Hammer, M. & 2007 & Harvard Business Review & 12 & & $\checkmark$ \\
\hline $\begin{array}{l}\text { Kettinger, W. J., \& Grov- } \\
\text { er, V. }\end{array}$ & 1995 & $\begin{array}{l}\text { Journal of Management Infor- } \\
\text { mation Systems }\end{array}$ & 21 & $A+/ 1$ & \\
\hline Morley, C. & 2004 & Paper presented at the Pre-ICIS & 17 & & \\
\hline $\begin{array}{l}\text { Sarkis, J., Presley, A., \& } \\
\text { Liles, D. }\end{array}$ & 1997 & $\begin{array}{l}\text { International Journal of Produc- } \\
\text { tion Economics }\end{array}$ & 13 & $A+/ 1$ & \\
\hline Véran, L. & 2006 & Comptabilité Contrôle Audit & 19 & $A / 2$ & \\
\hline $\begin{array}{l}\text { Vergidis, K., Turner, C. J., } \\
\text { \& Tiwari, A. }\end{array}$ & 2008 & $\begin{array}{l}\text { International Journal of Produc- } \\
\text { tion Economics }\end{array}$ & 13 & $A+/ 1$ & \\
\hline
\end{tabular}

\section{Tableau 1 : Liste des articles jugés significatifs}

\section{Analyse de contenu des sources documentaires}

Le travail consistant à étudier de manière approfondie des articles afin d'en identifier les concepts soulève les même difficultés que l'analyse d'entretiens, dans le cadre d'une étude qualitative : le chercheur dispose d'un corpus relativement important, duquel il doit réussir à extraire du sens (Gavard-Perret, Gotteland, Haon, \& Jolibert, 2008; Miles \& Huberman, 2003). Il s'agit en effet d'une analyse de données textuelles (de discours), pouvant faire l'objet d'une analyse de contenu ("une idée centrale dans l'analyse de contenu est que les nombreux mots du textes sont classés dans un nombre beaucoup plus petit de catégories", Weber, 1990, page 12), ou plus précisément d'une analyse thématique, dont le fonctionnement général est très proche de la précédente (Gavard-Perret et al., 2008). Les éléments identifiés dans le texte (Verbatim) devront être «codés », i.e. que de mots clés seront identifiés (dans un premier temps), puis utilisés pour « taguer» le texte.

\footnotetext{
${ }^{11}$ Classement AERES Juin 2009/CNRS Octobre 2007
} 


\section{$2.11^{\text {ère }}$ phase de codage}

L'analyse thématique d'un corpus permet "d'interpréter" son contenu (Fallery \& Rodhain, 2007). L'objectif, par rapport à notre problématique, était alors d'identifier un certain nombre de thématiques récurrentes dans la littérature portant sur le management des processus. Le logiciel QSR ${ }^{\circledR}$ NVivo 7 a été utilisé afin de coder les articles : "Le processus de codage consiste à découper le contenu d'un discours ou d'un texte en unités d'analyse (mots, phrases, thèmes...) et à les intégrer au sein de catégories sélectionnées en fonction de l'objet de recherche" (Thiétart, 2007, page 455).

Les unités d'analyse choisies ont été les phrases et/ou paragraphe. Le logiciel permet en effet d'importer les documents, de définir des mots clés ("nœuds", pour reprendre la terminologie utilisés dans le logiciel), puis d'affecter des phrases ou paragraphes des documents à un ou plusieurs de ces mots clés («Tag»). Par exemple, la phrase "Processes are structured sets of work activity that lead to specified business outcomes for customers" (Davenport \& Beers, 1995), a été codée sous les nœuds "processus" et "définition".

Il sera ensuite possible d'effectuer de requêtes plus ou moins complexes, du type : "quels sont les phrases/paragraphes qui abordent la notion de processus" ou encore "quels sont les phrases/paragraphes qui ont été codées sous les nœuds processus ET définition".

Il faut préciser que le codage a été effectué de manière émergente (codage "ouvert" pour reprendre la qualification de Strauss \& Corbin, 1990), au fur et à mesure de la lecture du corpus: Figure 7. L'objectif était ici de faire émerger les mots clés et thématiques, sans a priori.

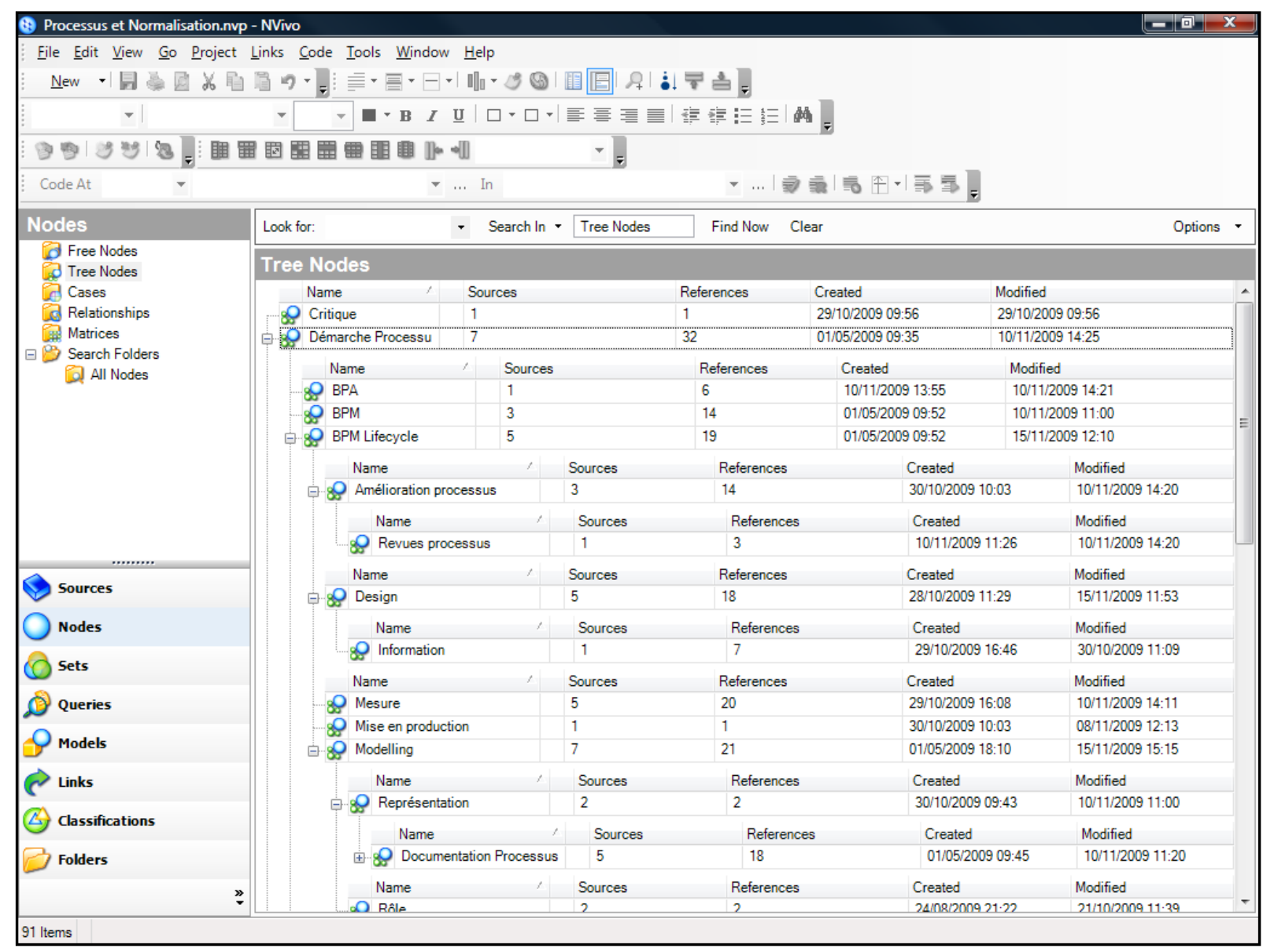

Figure 7 : Thématiques (Nouds) dans QSR ${ }^{\circledR}$ NVivo 7 


\subsection{Hiérarchisation des nœuds}

Les éléments du discours ont donc été catégorisés (Thiétart, 2007) : au terme du codage d'une dizaine d'articles à l'aide du logiciel, environ quatre-vingt mots clés (nœuds) ont été identifiés (Figure 8). Or ces nœuds manquaient de structuration d'une part (nécessité de regrouper les thématiques proches les unes des autres, par exemple) et, d'autre part, certains d'entre eux se recoupaient ou recouvraient les mêmes notions (exemple : "mise en production" et "exécution du processus" ou encore "Modelling" et "modèle"). La poursuite du codage devenait difficile : nécessité de faire le point sur les nœuds identifiés. L'utilisation d'un logiciel de cartographie heuristique, tel que Mind Manager ${ }^{\circledR}$ ou Free Mind, nous a semblé la plus appropriée pour procéder à cette analyse, l'objectif étant principalement de hiérarchiser les nœuds, hiérarchie qui pourra être reprise dans NVivo ("Tree Nodes"). Ce type d'application permet en effet de mettre en place des représentations des données sous une forme arborescente ; cette dernière, particulièrement visuelle, facilite le classement et l'organisation des informations.

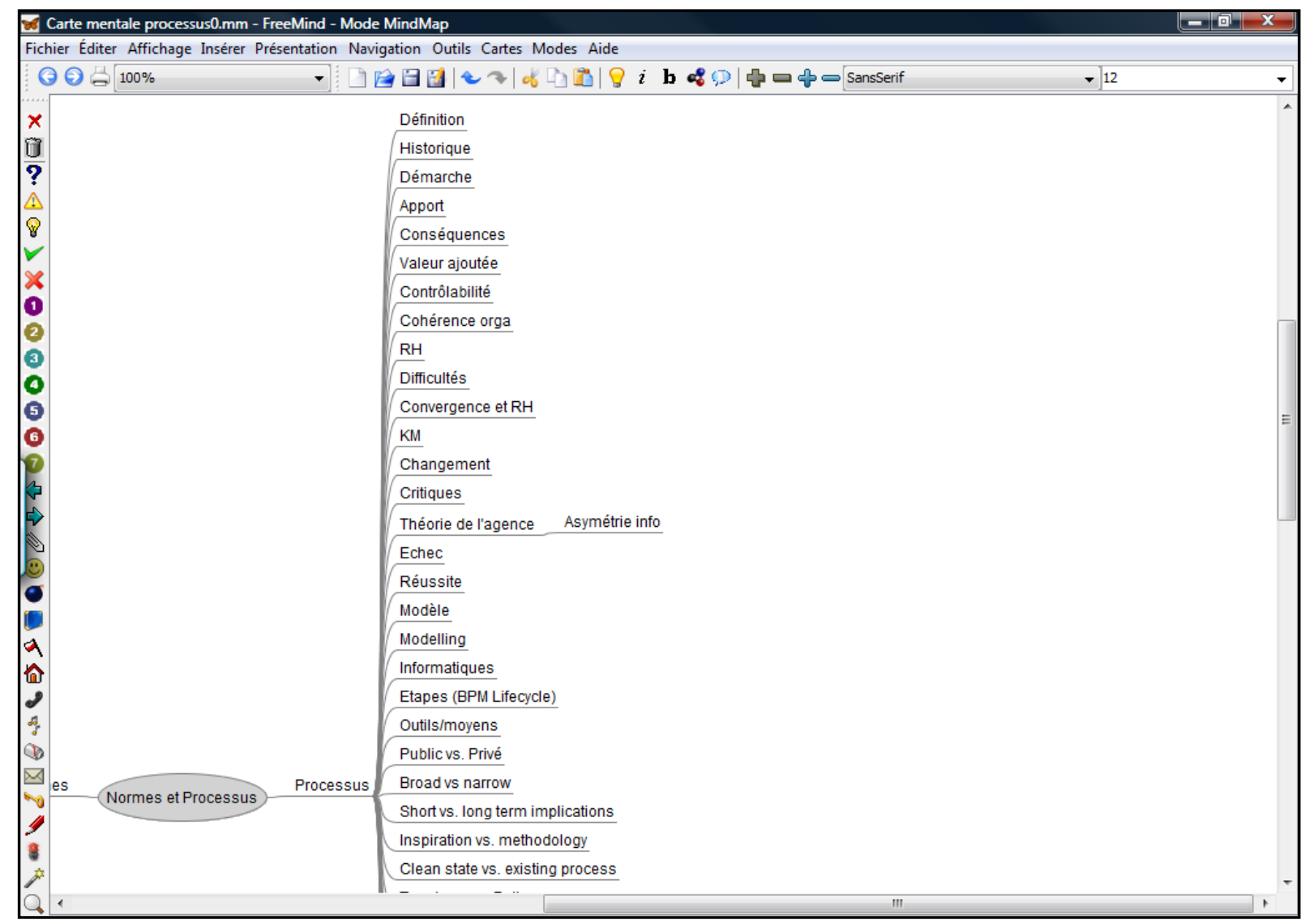

Figure 8 : Liste des nœuds identifiés dans QSR $^{\circledR}$ NVivo 7 , après exportation dans Free Mind

Un plan commence alors à se dégager à l'aide de la carte mentale ; il regroupe les 76 nœuds restants autour de cinq grandes thématiques : définition, stratégie, étapes, outils/moyens et conséquences (Figure 9). 


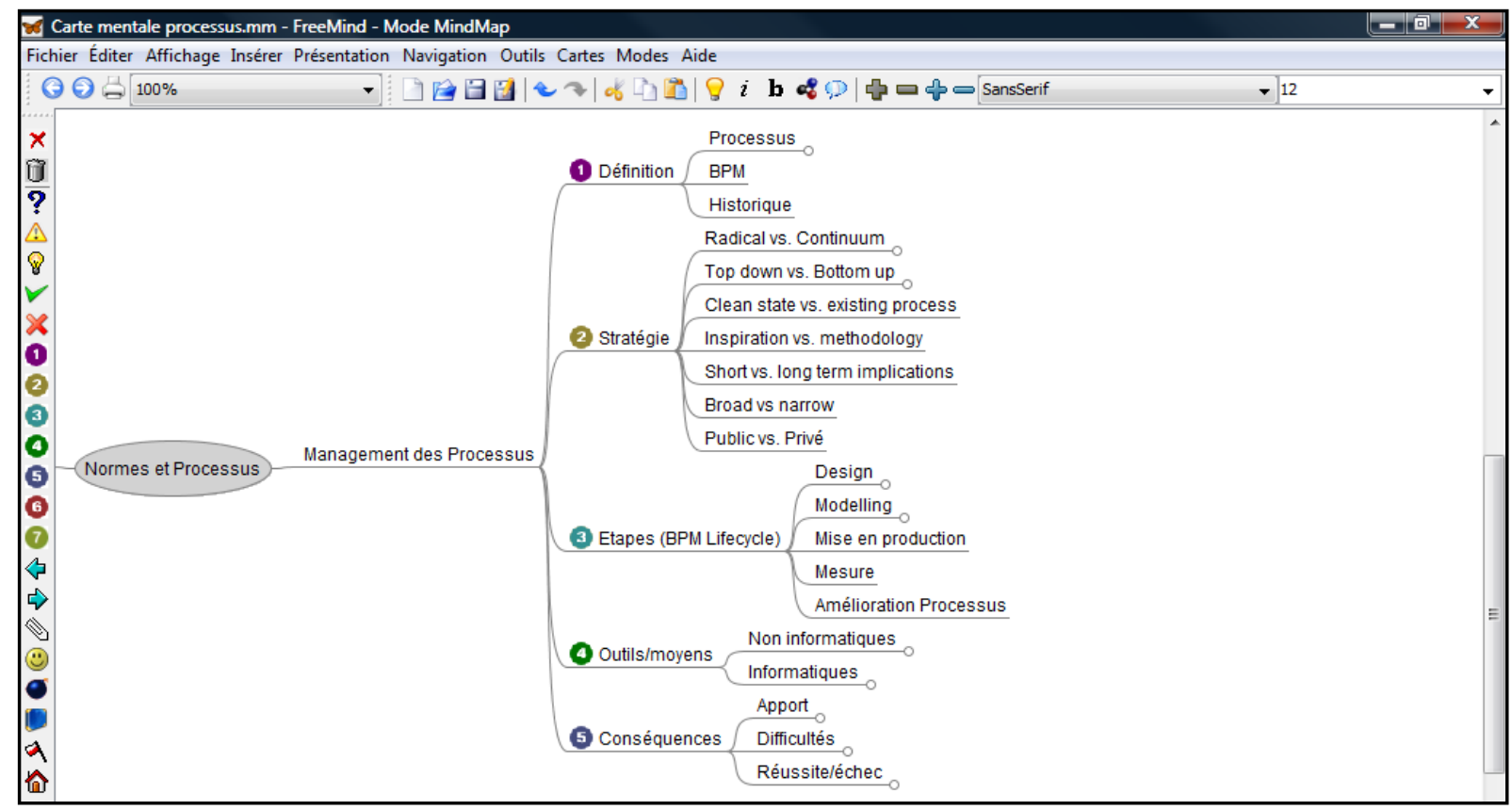

\section{Figure 9 : Grandes thématiques identifiées à l'aide du logiciel de cartographie heuristique}

Les nœuds, dans QSR ${ }^{\circledR}$ NVivo 7 ont alors été structurés en fonction de la hiérarchie mise en place dans FreeMind, afin de rationaliser et faciliter la suite du codage.

\section{$2.3 \quad 2^{\text {ème }}$ phase du codage}

Le codage des articles restants a rapidement fait apparaître un début de saturation : il n'y a plus de nouveaux nœuds identifiés lors du codage de nouveaux articles. Le graphique présenté dans la Figure 10 montre le nombre de nœuds identifiés (cumulés) sur l'axe des ordonnées, par rapport au nombre des articles codés, sur l'axe des abscisses. Ainsi, le $1^{\mathrm{er}}$ article codé a permis d'identifier 40 nœuds, le second, 21 de plus, soit un total de 61 nœuds au terme du codage de ce dernier, etc...

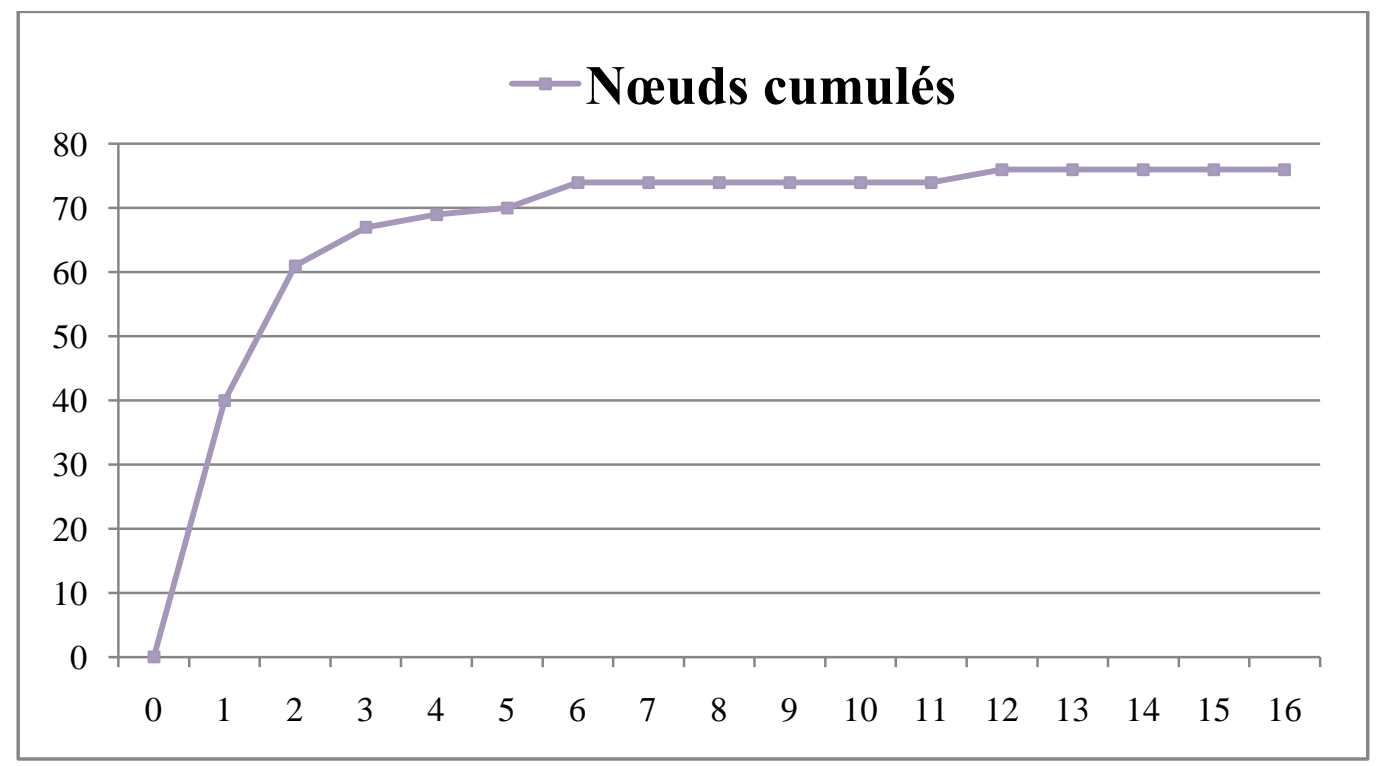

Figure 10 : Nouds cumulés par article codé 
Ce graphique montre qu'au terme du codage du $6{ }^{\text {ème }}$ article, 74 nœuds ont été identifiés et 76 à la fin du codage du $12^{\text {ème }}$ article; l'analyse des articles suivants n'a pas permis de mettre en évidence de nouveaux mots clés dans le domaine étudié. Il semble alors que la grande majorité des thématiques et mots clés sur le sujet aient été identifiés.

A ce stade de l'étude, les données ont été segmentées et "décontextualisées" (déconstruction), par le biais de leur catégorisation (Gavard-Perret et al., 2008; Savoie-Zajc, 2000; Tesch, 1990). Elles doivent à présent être "recontextualisées", "reconstruites", dans l'objectif de leur redonner du sens. Ici aussi, le logiciel de cartographie heuristique nous est apparu comme le plus approprié.

\section{Synthèse des sources documentaires}

Les données segmentées obtenues suite à l'analyse réalisée à l'aide du logiciel NVivo sont relativement nombreuses : plus de 700 verbatim identifiés au terme du codage des quinze articles. Ces données doivent être synthétisées, à l'aide du cadre des thématiques identifié lors de l'étape précédente (Figure 9). Le logiciel de cartographie heuristique permettra cette synthèse, dans la mesure où il donne une vue d'ensemble du travail effectué. Ce dernier s'effectuera alors en deux temps : développement, puis exportation de la carte.

\subsection{Développement de la carte heuristique}

Cette étape a consisté à développer la carte heuristique à l'aide des verbatim regroupés par nœud dans le logiciel d'analyse thématique : les requêtes, dans QSR ${ }^{\circledR}$ NVivo 7, permettent d'extraire tous les éléments codés se rapportant à un ou plusieurs mots clés. Or de très nombreux paragraphes codés comprennent des citations qui permettront à leur tour d'effectuer des recherches dans la bibliographie, via les références citées par les auteurs (qui seront retrouvés - ou rajoutés - dans le bloc-notes numérique et le gestionnaire de bibliographie).

Il est alors possible de procéder directement à la rédaction et au classement des idées, ainsi qu'à la mise en évidence des sources, dans le logiciel de cartographie heuristique : Figure 11.

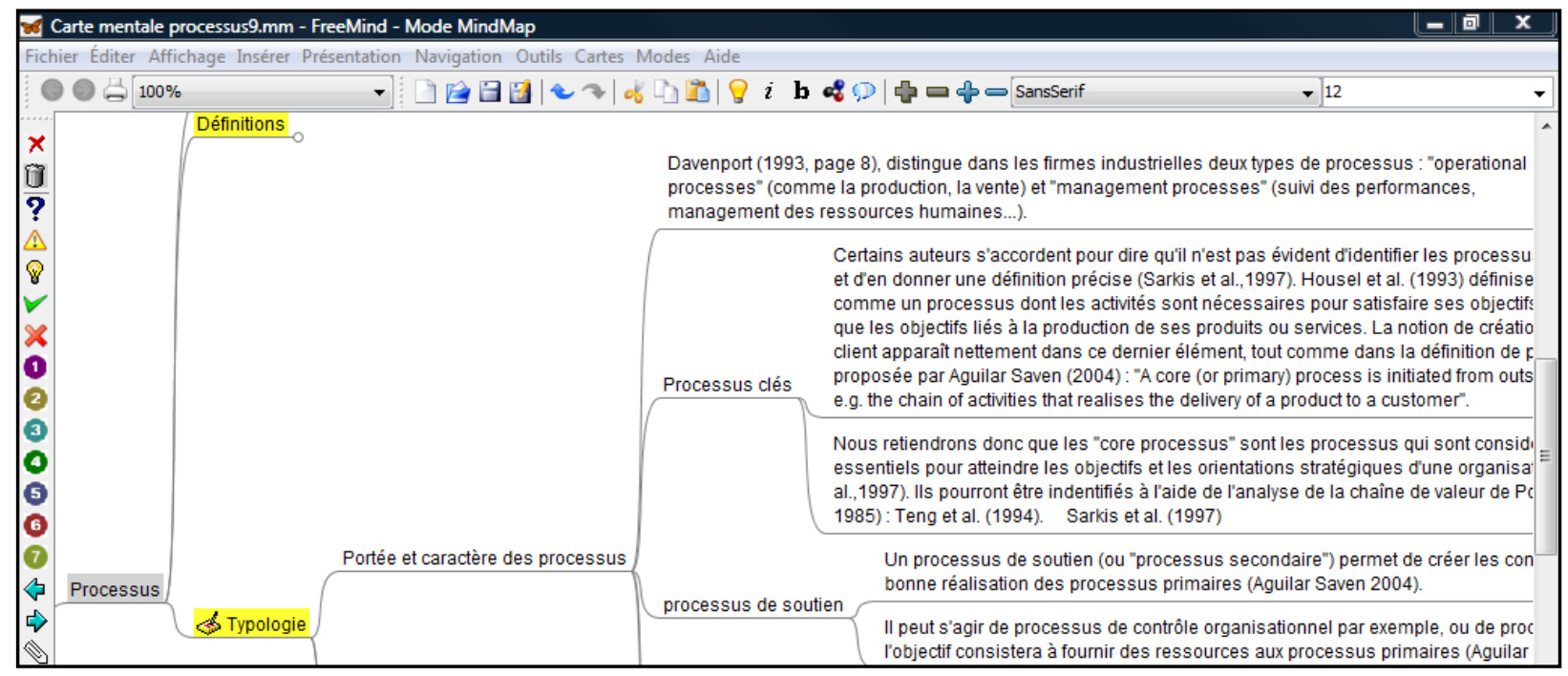

Figure 11 : Développement de la carte heuristique

La représentation sous forme d'arborescence offerte par ce type d'application facilite l'organisation des idées ainsi que l'identification d'éventuels problèmes de redondance ou d'enchâ̂nement des idées. Elle permettra la mise en cohérence de l'ensemble : choix des titres, rédaction des transitions, introductions et conclusions partielles. 


\subsection{Exportation de la carte heuristique dans un traitement de texte}

La carte mentale, une fois développée, peut être exportée sur un logiciel de traitement de texte, ici Microsoft ${ }^{\circledR}$ Word 2007. Ce dernier permettra une relecture et un travail sur la forme, mais surtout l'insertion d'illustrations et des références bibliographiques via le module complémentaire de EndNote Web (Figure 4).

Le chercheur pourra alors effectuer un retour sur la bibliographie à partir de ce document structuré et rédigé : la synthèse obtenue sera étoffée et approfondie, au gré des nouvelles lectures.

\section{Discussion}

Cette communication décrit la démarche qui a été celle d'un chercheur dans sa phase de découverte de son objet d'étude. Son intérêt majeur réside dans le fait qu'elle a permis à ce dernier de structurer un travail dont la complexité apparaissait, de prime abord, comme un obstacle difficile à contourner. Nous nous situons dans une problématique de construction de sens, dans l'acception développé par Weick (1995), à savoir une activité mentale permettant aux individus de structurer ce qu'ils perçoivent. Notre objectif était de créer d'une représentation des concepts contenus dans des articles académiques, avec la subjectivité qu'une telle démarche suppose. Les deux phases de «décontextualisation » et « recontextualisation » présentent en effet une grande subjectivité car elles sont le fruit de l'interprétation et de la réflexion du chercheur. Il ne s'agit en aucun cas d'un travail réalisé automatiquement par une application informatique : le logiciel aide le chercheur à structurer sa pensée. Ce travail se veut donc descriptif, et en aucun cas normatif.

Il soulève cependant un certain nombre de limites qu'il apparait indispensable de souligner. Tout d'abord, le choix et le nombre des articles "jugés significatifs" peut être discuté. Est-ce qu'un autre choix n'aurait pas abouti à des mots clés (nœuds) différents lors de la phase de codage ? Le fait que le codage de nouveaux articles n'ait pas permis la mise en évidence de nouveau nœuds nous amène cependant à penser que le résultat global obtenu serait sensiblement le même. Autre point : est-ce que le codage n'aurait pas dû porter sur l'ensemble du corpus ? Il semblerait que non : deux limites matérielles apparaissent rapidement. Tout d'abord, le codage d'un article d'une vingtaine de page nécessite entre 4 et 8 heures d'un travail particulièrement fastidieux. L'analyse d'une centaine d'articles semble alors concrètement difficile. Une seconde limite matérielle porte sur le nombre de verbatim obtenus, et leur utilisation ultérieure : le codage de quinze articles a mis en évidence un total de plus de 700 verbatim (phrases, paragraphes, ou partie de paragraphe), qui ont ensuite fait l'objet d'une analyse afin de permettre l'obtention de la synthèse finale. L'examen d'une centaine d'articles aurait alors conduit à un volume trop important de verbatim pour qu'une synthèse soit matériellement réalisable.

Une seconde limite porte sur le codage lui-même. L'analyse proposée s'inscrit dans le schéma des analyses qualitatives. Or, la qualité d'une analyse qualitative suppose deux caractéristiques (Weber, 1990) : la stabilité ("un codeur doit être capable d'obtenir, sur le même corpus, des résultats identiques plusieurs fois de suite", Gavard-Perret et al., 2008) et la reproductibilité ("il s'agit de voir dans qu'elle mesure les résultats de codage sont comparables entre deux codeurs", Gavard-Perret et al., 2008). Ces deux caractéristiques n'ont été encore été vérifiées : il s'agit d'une des futures pistes de travail de cette recherche.

Enfin, l'ensemble de la démarche permettra certes d'obtenir un document de travail pouvant servir de point de départ, mais il n'est qu'une étape dans le processus de réalisation d'un état de l'art. En effet, les références étudiées de façon approfondies ont permis, (1) d'identifier les 
grandes thématiques et de structurer leur contenu et (2) de trouver de nouvelles références et idées par le biais des travaux qu'elles citent, ces derniers permettant à leur tour d'effectuer des approfondissements dans les travaux cités, etc. La finalisation de cet état de l'art suppose une étape supplémentaire, à savoir un retour à la littérature : les recherches et lectures ultérieures permettrons de revenir sur certains points, de les approfondir, de les retravailler, et de les intégrer dans le cadre obtenu. Il s'agit donc avant tout d'une démarche permettant d'obtenir une synthèse facilitant le travail d'étude de la bibliographie par le chercheur.

\section{Conclusion}

Cette communication présente un retour d'expérience sur l'apport méthodologique de différents outils de classification de données qualitatives. Ce travail a pour but d'apporter des pistes de réponses aux nombreuses interrogations que le chercheur peut se poser. Il ne s'agit cependant pas d'une présentation normative : l'objectif était de décrire, de la façon la plus objective possible, le travail qui a été effectué, et de faire connaître les apports de certaines applications en matière de recherche en sciences de gestion.

En effet, les outils informatiques tels que les bloc-notes numériques, gestionnaires de bibliographie, application de cartographie heuristique, analyse thématiques... peuvent apporter une aide précieuse au chercheur. Leur usage va s'imposer dans les prochaines années: ils apportent une réponse aux difficultés soulevées par le traitement et l'analyse d'une profusion de sources qui seront de plus en plus systématiquement numérisées. Il est cependant dommage de devoir mobiliser au moins cinq applications distinctes, au lieu d'une ou deux...

Ce travail montre comment ces outils complémentaires peuvent être combinés dans un objectif précis, à savoir l'établissement d'un état de l'art. Il peut faire l'objet de deux lectures différentes. Tout d'abord, dans sa globalité : l'ensemble de la démarche aura surtout une utilité dans le cas de la découverte d'un nouvel objet d'étude (cas d'un jeune chercheur souhaitant délimiter et identifier les grandes thématiques de son objet d'étude, ou d'un chercheur souhaitant étendre son domaine à un sujet dont il a peu de connaissances). Une autre lecture pourra consister à n'utiliser une partie de la démarche, comme la gestion documentaire par exemple.

Enfin, il est important de souligner que la démarche présentée ici n'est pas fondamentalement différente de celle qui pourrait être celle d'un chercheur ne mobilisant pas les outils informatiques pour réaliser son état de l'art. Le principe général reste la recherche d'informations dans la littérature, leur analyse et leur synthèse. Elle présente néanmoins l'intérêt majeur de permettre la rationalisation du traitement des informations réunies et de faciliter ces phases d'analyse et de synthèse : accès beaucoup plus rapide et efficace aux données. Elle réduit aussi de manière plus que significative le volume des documents papiers à mobiliser : dans une optique mettant de plus en plus en avant les préoccupations environnementales, la recherche "zéro papier" devient réalité...

\section{Bibliographie}

Alami, S., Desjeux, D., Garabuau-Moussaoui, I. (2009). Les méthodes qualitatives: Collection Que sais-je?

Adamides, E. D., \& Karacapilidis, N. (2006). "A Knowledge Cendred Framework for Collaborative Business Process Modelling", Business Process Management Journal, Vol. 12 (5): 557-575.

Aguilar-Saven, R. S. (2004). "Business process modelling: Review and framework". International Journal of Production Economics, 90(2): 129-149.

Aytulun, S. K., \& Guneri, A. F. (2008). "Business process modelling with stochastic networks". International Journal of Production Research, 46(10): 2743-2764. 
Bandara, W., \& Rosemann, M. (2005). "What Are the Secrets of Successful Process Modeling? Insights From an Australian Case Study". Systèmes d'Information et Management, 10(3): 47-68.

Chan, S., \& Choi, C. (1997). "A Conceptual and Analytical Framework for Business Process Re-engineering". International Journal of Production Economics, 50: 211-223.

Chatha, K. A., Ajaefobi, J. O., \& Weston, R. H. (2007). "Enriched multi-process modelling in support of the life cycle engineering of Business Processes". International Journal of Production Research, 45(1): 103-141.

Davenport, T. H., \& Beers, M. C. (1995). "Managing Information about Processes". Journal of Management Information System, 12(1): 57-80.

Dennis, A. R., Carte, T. A., \& Kelly, G. G. (2003). "Breaking the Rules : Success and Failure in Groupware-Supported Business Process Reengineering". Decision Support Systems, 36(1): 31

Fallery, B., \& Rodhain, F. (2007). "Quatre approches pour l'analyse de données textuelles : lexicale, linguistique, cognitive et thématique". Paper presented at the XVIème Conférence de l'Association Internationale de Management Stratégique (AIMS) 7-9 juin 2007, Montréal, Canada.

Gavard-Perret, M. L., Gotteland, D., Haon, C., \& Jolibert, A. (2008). Méthodologie de la recherche : réussir son mémoire ou sa thèse en science de gestion: Pearson Education.

Hammer, M. 1990. "Reengineering Work : Don't Automate, Obliterate". Harvard Business Review, Juillet-Août: 104-112.

Hammer, M. (2007). "The Process Audit". Harvard Business Review, 85(4): 111-123 (113 pages).

Kettinger, W. J., \& Grover, V. (1995). "Special Section: Toward a Theory of Business Process Change Management". Journal of Management Information Systems, 12(1): 9-30.

Miles, M. B., \& Huberman, A. M. (2003). Analyse des données qualitatives. Bruxelles: De Boeck.

Morley, C. (2004). "Un cadre unificateur pour la représentation des processus". Paper presented at the Pre-ICIS, déc.

Sarkis, J., Presley, A., \& Liles, D. (1997). "The strategic evaluation of candidate business process reengineering projects". International Journal of Production Economics, 50(23): 261-274.

Savoie-Zajc, L. (2000). "L'analyse de données qualitatives : pratiques traditionnelle et assistée par le logiciel NUD-IST". Recherches qualitatives, 21: 99-123.

Strauss, A., \& Corbin, J. (1990). Basics of qualitative research : grounded theory procedures and techniques. Londres: Sages.

Tesch, R. (1990). Qualitative Research: An analysis Types and Software Tools. New York: New York : The Falmer Press.

Thiétart, R.-A. (2007). Méthodes de recherche en management: Dunod.

Vergidis, K., Turner, C. J., \& Tiwari, A. (2008). "Business process perspectives: Theoretical developments vs. real-world practice". International Journal of Production Economics, 114(1): 91-104.

Véran, L. (2006). "Activités et processus, modélisation gestionnaire et comportements des acteurs". Comptabilité Contrôle Audit, 12(1): 65-84.

Weber, R. P. (1990). Basic content analysis. Newbury Park: Sage Publications.

Weick, K. E. (1995). Sensemaking in organizations. Sage Publications. 